\title{
Study on the Misunderstanding of Sports Functional Beverage
}

\author{
Wu Yue \\ Sport Department, Huanggang Normal University, Huanggang 438000, China
}

\begin{abstract}
With the development of the social economy and the rise of the national fitness campaign, the development of sports functional beverage has become a part of sports consumption. Sports functional beverage has significant effect on improving athletic ability and fast recovery. But the inappropriate choice of beverage and drinking arrangements will cause irreparable damage to the body. Only the scientific and rational drinking, sports functional beverage will really play its whole function.
\end{abstract}

Key words: Sports functional beverage, national fitness, sports consumption, China.

\section{Introduction}

With the increasing of public demand for fitness activities, spending for sports consumption is also significantly rising. These consumptions include sports equipment, sports apparel, event tickets and other expenses, and also including the consumption of sports functional beverage. "Sleepy and tired, drink functional beverages!” This has become a classic advertisement for beverage manufacturers. But for the public fitness people, functional beverage, which could fast replenish energy and maintain the body's electrolyte balance, has become the preferred thing in sports fitness events.

At present, the international sports functional beverage market is nearly 100 billion dollars and the global per capita consumption of functional beverage has reached $18 \mathrm{~kg}$ [1]. But in our country, the functional beverage market has just grown up, the per capita consumption of functional beverage is only $4 \mathrm{~kg}$ in nowadays, which is the $1 / 4$ world's average level. At the same time, there is a certain misunderstanding of the sports function beverage.

Corresponding author: Wu Yue, research field: anti-aging experiment.

\section{Misunderstanding of Sports Functional Beverage}

\subsection{The Wrong Choice of Beverage}

The functional beverage is a large class of beverage including sports drinks, energy drinks and other special functional drinks (such as adding vitamin and mineral nutritional beverage and herbal ingredients in the herbal beverage etc.), and provide specific nutrition and health function for special people. In recent years, there are lots of beverage which under the banner of "function" spewing out, mainly including Mizone, Activation, Screaming as representative of the nutritional beverage; Gatorade, fresh, SUPER ULTIMA as representatives of sports drinks; Red Bull, Lucozade, Lipovitan, Ichimore, V Guarana Energy Drink as representatives of the energy drinks.

With the variety of human body's movement and the movement environment, we have different requirements on beverage during exercise. Take the sports drink as an example, it contains sodium, potassium, calcium, magnesium and other ingredients, which only suitable after sports, in order to supplement the missing part of the body electrolyte. If you take it as a daily drink, it will be the wrong choice. Appropriate proportion of sugar content in the sports beverage is $6 \%-7 \%$, which not only ensure human 
mostly absorption, but also prevent transient rising of blood glucose [2]. Some of the functional beverage has high level of sugar content, which could lead diuretic effect for the human body, accelerate kidney discharge water, instead of thirst quenching effect, it will lead the body more and more dry which beyond our desire. For people with diabetes, hypertension and obesity, whether they could choose functional beverage or not, need process a lot of consideration.

\subsection{Misunderstanding on Drinking Time}

There is distinction on when we take functional beverage. If you do not have lots of exercise with time less than $1 \mathrm{~h}$, then instead of taking functional sports beverage, we recommend you to drink some salt water or natural mineral water to supplement the body. If you take the relatively large amount of exercise with the time is longer than $1 \mathrm{~h}$, a lot of human sweat (take playing basketball, football, badminton etc.,) with sweat a sportswear, then drinking sports beverage can have full play to the its role. If you take about 300 milliliters of sports beverage before $1 \mathrm{~h}$ exercise, it will advance promotion of the synthesis of glycogen. Drinking 100-200 mL every 15-20 min during exercise, can add sugar, improve exercise capacity. If you take $500 \mathrm{~mL}$ within one hour after exercise, it can restore physical strength.

Different sports items have different functional consumption, so we have different demands in body recovery and nutrition needs. For example, if we added sugar beverage before and during the football game, it can save muscle glycogen and delay the occurrence of fatigue. And strength exercises have high requirements on human power and coordination of nerves and muscles, so movement of creating supplement can increase the body phosphocreatine reserve content [3].

\subsection{Misunderstanding on Quantity of Drinking}

Different groups of people need to be careful on drinking sports beverage. Currently, there is increasing proportion of children to drink sports beverage year by year, but it could not bring satiety. If children continue to absorb other physical energy, it would be easy lead induce potential disease of type 2 diabetes and metabolism syndrome and overweight [4-5]. Meanwhile, it can also lead increasing of serum uric acid levels, which is closely related to the cardiovascular disease of adults. If you drink beverages which include large amounts of caffeine before and after exercise, it will lead to sudden heart disease or instability heart rate. If you are potential heart patient, the harm will be greater.

Needs to be mentioned that the various nutrients prime number and proportion in the body are fixed. If you take too much or too little of them will destroy the body's nutritional balance state. Therefore, when the body is not the lack of certain nutrients, artificially increased a large number of the nutrient prime will disrupt the balance, which not only useless for health, but increase the burden for the body and destroy the health. Drinking sport beverages for long term may affect sleep, vitamin poisoning, damage dental, malnutrition and many other issues [6]. It is recommended that the amount of sport beverage should not exceed $300 \mathrm{~mL}$ in everyday.

\section{Conclusions}

China has large population with vast sports consumer market. Under the overall background of the rapid development of sports industry, sports beverage has become an important part of the exercise. For the different groups of people, it is very important to know how to scientifically choose drinks, arrange for the drinking time, monitor the quantity of drinking. Only scientific and rational use of functional beverage, can really make it play its wholly role in the national fitness.

\section{References}

[1] Coyle, E. F. 2004. "Fluid and Fuel Intake during Exercise [J].” J. Sports Sci. 22: 39-55.

[2] Gentry, L., Carmen, P., and Barry, M. P. 2011. 
"Beverage Patterns and Trends among School Aged Children in the US, 1989-2008 [J].” Nutrition Journal 10: 103.

[3] Malik, V. S., Popkin, B. M., and Bray, G. A., et al. 2010. "Sugar Sweetened Beverages and Risk of Metabolic Synd-rome and Type 2 Diabetes: Ameta-anaysis [J]." Diabetes Care 33 (11): 2477-83.

[4] Rehrer, N. 2001. "Fluid and Electrolyte Balance in
Ultra-endurance Sport [J].” Sports Med. 31: 701-15.

[5] el-Sayed, M. S., Mac, L. D., and Rattu, A. J. 1997. "Exogenous Carbohydrate Utilisation: Effects on Metabolism and Exercise Performance [J].” Comp. Biochem. Physiol. 118 (3): 789-803.

[6] Shi, X., and Gisolfi, C. V. 1998. "Fluid and Electrolyte Replacement during Intermittent Exercise [J].” Sports Med. 25: 157-72. 